\title{
COMMUTATOR APPROXIMANTS
}

\author{
P. J. MAHER
}

(Communicated by Paul S. Muhly)

\begin{abstract}
This paper deals with minimizing $\|B-(A X-X A)\|_{p}$, where $A$ and $B$ are fixed, $B \in \mathscr{C}_{p}$, and $X$ varies such that $A X-X A \in \mathscr{C}_{p}$. (Here, $\mathscr{C}_{p}$ denotes the von Neumann-Schatten class and $\|\cdot\|_{p}$ denotes its norm.) The main result (Theorem 3.2) says that if $A$ is normal and $A B=B A$ then $\|B-(A X-X A)\|_{p}, 1 \leq p<\infty$, is minimized if and for $1<p<\infty$ only if, $A X-X A=0$; and that the map $X \rightarrow\|B-(A X-X A)\|_{p}^{p}, 1<p<\infty$, has a critical point at $X=V$ if and only if $A V-V A=0$.
\end{abstract}

\section{INTRODUCTION}

A well-known result of Halmos [6, Problem 233; 4] says that if $A$ (or $B$ ) commutes with $A B-B A$ then

$$
\|\alpha I-(A B-B A)\| \geq\|\alpha I\| .
$$

The related inequality (1.2) was obtained by Anderson [2, Theorem 1.7] who showed that if $A$ is normal and commutes with $B$ then, for all $X$ in $\mathscr{L}(H)$,

$$
\|B-(A X-X A)\| \geq\|B\| .
$$

In this paper we obtain an inequality similar to $(1.2)$ where the operator norm is replaced by the $\|\cdot\|_{p}$ norm on the von Neumann-Schatten classes $\mathscr{C}_{p}$, $1 \leq p<\infty$. This inequality, contained in Theorem 3.2(a), says that if the normal operator $A$ commutes with $B$, where $B \in \mathscr{C}_{p}$, and if $X$ varies such that $A X-X A \in \mathscr{C}_{p}$ then, for $1 \leq p<\infty$,

$$
\|B-(A X-X A)\|_{p} \geq\|B\|_{p}
$$

with equality occurring, and for $1<p<\infty$ only if $A X-X A=0$. Thus in Halmos' terminology [5] the zero commutator is the commutator approximant in $\mathscr{C}_{p}$ of $B$.

Additionally, we classify the critical points of the map $F_{p}$, on $\mathscr{S}=\{X$ : $\left.A X-X A \in \mathscr{C}_{p}\right\}$, defined by

$$
F_{p}: X \rightarrow\|B-(A X-X A)\|_{p}^{p}
$$

Received by the editors March 21, 1990 and, in revised form, January 7, 1991.

1980 Mathematics Subject Classification (1985 Revision). Primary 47B47, 47A30; Secondary 47B10.

Key words and phrases. Commutator, von Neumann-Schatten class, Fuglede's theorem, functional calculus. 
(that is, we classify $\left\{\mathrm{V}\right.$ : the Fréchet derivative $\left.D_{V} F_{p}=0\right\}$ ). The local result, Theorem 3.2(b), says that under the same hypotheses $(A$ normal and $A B=B A$ ) $V$ is a critical point of $F_{p}, 1<p<\infty$, if and only if $A V-V A=0$.

Note that $\alpha I-(A X-X A)$ cannot be compact with $A$ and $X$ bounded. (This follows from the Wielandt/Wintner result $[9,10]$ that a commutator of bounded operators cannot equal the identity. If $\alpha I-(A X-X A)$ were compact, then in the Calkin algebra, the identity would be the commutator of the images of $A$ and $X$ contradicting Wielandt/Wintner in the context of normed algebras with unit.) Hence, in infinite dimensions there is no question of minimizing $\|\alpha I-(A X-X A)\|_{p}$.

\section{Preliminaries}

Let $H$ denote a separable complex Hilbert space and $\mathscr{L}(H)$ denote the space of all bounded linear operators mapping $H$ into itself. For details of the von Neumann-Schatten classes $\mathscr{C}_{p}$ and norms $\|\cdot\|_{p}$, see [3, Chapter XI; 8, Chapter 2]. We state below the Aiken, Erdos, and Goldstein differentiation result. The real part of a complex number $z$ is denoted by $\mathscr{R} z$.

Theorem 2.1 [1, Theorem 2.1]. Let the map $\Phi: \mathscr{C}_{p} \rightarrow \mathbb{R}^{+}$be given by $\Phi: X \rightarrow$ $\|X\|_{p}^{p}$. Then

(a) for $1<p<\infty$, the map $\Phi$ is differentiable at every $X$ in $\mathscr{C}_{p}$ with derivative $D_{X} \Phi$ given by

$$
\left(D_{X} \Phi\right)(S)=p \mathscr{R} \tau\left[|X|^{p-1} U^{*} S\right],
$$

where $\tau$ denotes trace, $X=U|X|$ is the polar decomposition of $X$, and $S \in \mathscr{C}_{p}$;

(b) for $0<p \leq 1$, provided $\operatorname{dim} H<\infty$, the same result holds at every invertible element $X$.

\section{On Minimizing $\|B-(A X-X A)\|_{p}$}

The proof of the local result, Theorem 3.2(b), depends on Lemma 3.1, which is a variation of the well-known Kleinecke-Shirokov theorem [6, Problem 232].

Lemma 3.1. Let $A$ be normal and commute with $A V-V A$. Then $A V-V A$ $=0$.

Proof. The proof hinges on the spectral resolution of $A$. This says that there exists a spectral measure $E(\cdot)$ such that, for each $\varepsilon>0$, there exist disjoint Borel sets $\Delta_{i}, 1 \leq i \leq N$, with the property that

$$
\text { if } \lambda_{i} \in \Delta_{i} \text { and } S=\sum_{i=1}^{N} \lambda_{i} E\left(\Delta_{i}\right) \text { then }\|A-S\|<\varepsilon .
$$

The operator $A V-V A$ commutes with $A$ and hence with each of the spectral projections $E\left(\Delta_{i}\right)$, and so, since $\sum_{i=1}^{N} E\left(\Delta_{i}\right)=E\left(U \Delta_{i}\right)=I$,

$$
A V-V A=(A V-V A) \sum_{i=1}^{N} E\left(\Delta_{i}\right)=\sum_{i=1}^{N} E\left(\Delta_{i}\right)(A V-V A) E\left(\Delta_{i}\right) .
$$

Since the Borel sets are pairwise disjoint, $E\left(\Delta_{i}\right) E\left(\Delta_{j}\right)$ equals $E\left(\Delta_{i}\right)$ if $i=j$ and is zero if $i \neq j$. Hence on substituting for $S$, we find that for each (fixed) 
$i$ we have $E\left(\Delta_{i}\right)(S V-V S) E\left(\Delta_{i}\right)=0$. So,

$$
\begin{aligned}
\left\|E\left(\Delta_{i}\right)(A V-V A) E\left(\Delta_{i}\right)\right\| & =\left\|E\left(\Delta_{i}\right)[(S V-V S)+(A-S) V-V(A-S)] E\left(\Delta_{i}\right)\right\| \\
& \leq 2\|A-S\|\|V\|\left\|E\left(\Delta_{i}\right)\right\|<2 \varepsilon\|V\| .
\end{aligned}
$$

Since, from (1), $\|A V-V A\|=\sup \left\|E\left(\Delta_{i}\right)(A V-V A) E\left(\Delta_{i}\right)\right\|$, then $A V-V A$ $=0$.

Theorem 3.2. Let $A$ be normal, $A B=B A$, and $B$ be in $\mathscr{C}_{p}$. Let $\mathscr{S}=\{X$ : $\left.A X-X A \in \mathscr{C}_{p}\right\}$ and $F_{p}: \mathscr{S} \rightarrow \mathbb{R}^{+}$be given by

$$
F_{p}: X \rightarrow\|B-(A X-X A)\|_{p}^{p} .
$$

Then

(a) for $1 \leq p<\infty$, the map $F_{p}$ has a global minimizer at $V$ if, and for $1<p<\infty$ only if, $A V-V A=0$;

(b) for $1<p<\infty$, the map $F_{p}$ has a critical point at $V$ if and only if $A V-V A=0$;

(c) for $0<p \leq 1$, the map $F_{p}$ has a critical point at $V$ if $A V-V A=0$ provided $\operatorname{dim} H<\infty$ and $B-(A V-V A)$ is invertible.

Proof. (a) The idea is to replace $B$ by the compact, normal operator $|B|$. Let $B=U|B|$ be the polar decomposition of $B$ so that $\operatorname{Ker} U=\operatorname{Ker}|B|$ and $|B|=U^{*} B\left(\in \mathscr{C}_{p}\right)$. Since $U$ is a partial isometry so is $U^{*}$ (so that $\left\|U^{*}\right\|=1$ ). As $\left\|U^{*} T\right\|_{p} \leq\left\|U^{*}\right\|\|T\|_{p}=\|T\|_{p}$ for arbitrary $T$ in $\mathscr{C}_{p}$, then

$$
\begin{aligned}
\|B-(A X-X A)\|_{p}^{p} & \geq\left\||B|-U^{*}(A X-X A)\right\|_{p}^{p} \\
& \geq \sum_{n}\left|\left\langle\left[|B|-U^{*}(A X-X A)\right] \phi_{n}, \phi_{n}\right\rangle\right|^{p}=\sum,
\end{aligned}
$$

say, for an arbitrary orthonormal basis $\left\{\phi_{n}\right\}$ of $H$ (the last inequality following from [8, Lemma 2.3.4]).

Because $A B=B A$ and $A$ is normal, by Fuglede's theorem, we have $A B^{*}=$ $B^{*} A$, and hence $A|B|^{2}=|B|^{2} A$. Moreover, by the functional calculus [8, Theorem 1.7.7(vi)], $A|B|=|B| A$ (and, indeed, $A|B|^{p-1}=|B|^{p-1} A$ ). Therefore, there exists an orthonormal basis $\left\{\xi_{k}\right\} \cup\left\{\psi_{m}\right\}$ of $H$ such that $\left\{\psi_{m}\right\}$ is an orthonormal basis of $\operatorname{Ker}|B|$ and $\left\{\xi_{k}\right\}$ consists of common eigenvectors of $A$ and $|B|$. (I thank the referee for suggesting this basis.) Hence, $\sum_{k}\left\langle|B| \xi_{k}, \xi_{k}\right\rangle^{p}=\|B\|_{p}^{p}$. As $B^{*} A=A B^{*}$ and $A|B|=|B| A,|B| U^{*} A=$ $A|B| U^{*}=|B| A U^{*}$. In (1), take $\left\{\phi_{n}\right\}=\left\{\xi_{k}\right\} \cup\left\{\psi_{m}\right\}$. If $\phi_{n}=\xi_{k}$, then $\left\langle U^{*} A X \xi_{k}, \xi_{k}\right\rangle=\left\langle A U^{*} X \xi_{k}, \xi_{k}\right\rangle$ and hence, as $\xi_{k}$ is also an eigenvector of the normal operator $A,\left\langle U^{*}(A X-X A) \xi_{k}, \xi_{k}\right\rangle=\left\langle\left(A U^{*} X-U^{*} X A\right) \xi_{k}, \xi_{k}\right\rangle=0$. Thus (1) becomes

$$
\begin{aligned}
\sum & =\sum_{k}\left\langle|B| \xi_{k}, \xi_{k}\right\rangle^{p}+\sum_{m}\left|\left\langle U^{*}(A X-X A) \psi_{m}, \psi_{m}\right\rangle\right|^{p} \\
& \geq \sum\left\langle|B| \xi_{k}, \xi_{k}\right\rangle^{p}=\|B\|_{p}^{p}
\end{aligned}
$$

as desired.

For $1<p<\infty$ the uniqueness assertion follows from the convexity of the set $\mathscr{S}=\left\{X: A X-X A \in \mathscr{C}_{p}\right\}$.

(b) Let $V$ be in $\mathscr{S}$ so that $B-(A V-V A) \in \mathscr{C}_{p}$. Let $S$ be arbitrary subject to the condition that $B-(A(V+S)-(V+S) A) \in \mathscr{C}_{p}$, that is, $S A-A S \in \mathscr{C}_{p}$. 
Let $\Psi: X \rightarrow B-(A X-X A)$ and $\Phi: X \rightarrow\|X\|_{p}^{p}$. Then $F_{p}=\Phi \circ \Psi$. As $F_{p}$ is real-valued, the Fréchet derivative of $F_{p}$ at $V$, denoted by $D_{V} F_{p}$, is given by

$$
\left(D_{V} F_{p}\right)(S)=\lim _{h \rightarrow 0} \frac{F_{p}(V+h S)-F_{p}(V)}{h} .
$$

From this it follows that

$$
\left(D_{V} F_{p}\right)(S)=\left(D_{B-(A V-V A)} \Phi\right)(S A-A S) .
$$

Let $V$ be a critical point of $F_{p}$ so that $\left(D_{V} F_{p}\right)(S)=0$ for all $S$ in $\mathscr{S}$. Let $B-(A V-V A)=U_{1}|B-(A V-V A)|$ be the polar decomposition of $B-(A V-V A)$. Then from Theorem 2.1 and (2),

$$
0=p \mathscr{R} \tau\left[|B-(A V-V A)|^{p-1} U_{1}^{*}(S A-A S)\right]=p \mathscr{R} \tau[Y(S A-A S)],
$$

where $Y=|B-(A V-V A)|^{p-1} U_{1}^{*}$. Take $S=f \otimes g$, where $f$ and $g$ are arbitrary vectors in $H$. (The rank one operator $x \rightarrow\langle x, f\rangle g$, where $x \in H$, is denoted $f \otimes g$. Note that $\tau[T(f \otimes g)]=\langle T g, f\rangle$, cf. [8, pp. 73, 90].) Then, as $S \in \mathscr{C}_{1}$ (whence $Y S \in \mathscr{C}_{1}$ ), from the invariance of trace [8, Theorem 2.2.4(iv)] we have $\tau[Y S A]=\tau[A Y S]$. Thus, from (3)

$$
0=\mathscr{R} \tau[(A Y-Y A) S]=\mathscr{R}\langle(A Y-Y A) g, f\rangle .
$$

Because $f$ and $g$ are arbitrary, $A Y-Y A=0$, that is,

$$
A|B-(A V-V A)|^{p-1} U_{1}^{*}=|B-(A V-V A)|^{p-1} U_{1}^{*} A .
$$

We claim that if

$$
A|B-(A V-V A)| U_{1}^{*}=|B-(A V-V A)| U_{1}^{*} A,
$$

then the assertion that $A V-V A=0$ will be proved. For suppose (6) holds. Then taking adjoints and using the polar decomposition of $B-(A V-V A)$ and Fuglede's theorem (which gives $B A^{*}=A^{*} B$ ), we get $(A V-V A) A^{*}=$ $A^{*}(A V-V A)$. By Fuglede again, we get $(A V-V A) A=A(A V-V A)$. Hence, by Lemma 3.1, $A V-V A=0$.

Proof of (6). Write $Z=|B-(A V-V A)|^{p-1}$. Then (5) says that

$$
A Z U_{1}^{*}=Z U_{1}^{*} A
$$

and (6) is the same as $A Z^{1 /(p-1)} U_{1}^{*}=Z^{1 /(p-1)} U_{1}^{*} A$. This will follow by the functional calculus (cf. [7, Theorem 4.1]) from

$$
A Z^{n} U_{1}^{*}=Z^{n} U_{1}^{*} A
$$

for the function $f: t \rightarrow t^{1 /(p-1)}, 1<p<\infty$, where $t \in \mathbb{R}^{+} \supseteq \sigma(Z)$ can be approximated uniformly by a sequence $\left(q_{i}\right)$ of polynomials without constant term (for $f(0)=0)$. Thus, (8) will imply that $A q_{i}(Z) U_{1}^{*}=q_{i}(Z) U_{1}^{*}$ and hence that $A Z^{1 /(p-1)} U_{1}^{*}=Z^{1 /(p-1)} U_{1}^{*} A$.

To prove (8) we use induction. We need the following assertion: $A Z=Z A$. To prove this assertion, note that in the polar decomposition of $B-(A V-V A)$ we have $\operatorname{Ker} U_{1}=\operatorname{Ker}|B-(A V-V A)|=\operatorname{Ker} Z$ (by the spectral theorem) so that $\left(\operatorname{Ker} U_{1}\right)^{\perp}=\operatorname{Ran} Z^{-}$. Thus, $U_{1}^{*} U_{1}$, the projection onto $\left(\operatorname{Ker} U_{1}\right)^{\perp}$, satisfies $U_{1}^{*} U_{1} Z=Z$ and hence $Z U_{1}^{*} U_{1} Z=Z^{2}$. Now take adjoints of (7): then $U_{1} Z A^{*}=A^{*} U_{1} Z$ and hence, by Fuglede, $U_{1} Z A=A U_{1} Z$. Then by (7)

$$
A Z^{2}=A Z U_{1}^{*} U_{1} Z=Z U_{1}^{*} A U_{1} Z=Z U_{1}^{*} U_{1} Z A=Z^{2} A .
$$


Taking positive square roots of $Z^{2}$ [8, Theorem 1.7.7(vi)] we get $A Z=Z A$. Returning now to (8): for $n=1,(8)$ is just (7); whilst the inductive step is now immediate from $A Z=Z A$.

Conclusion so far: $V$ is a critical point of $F_{p} \Rightarrow A V-V A=0$.

Conversely, let $V$ satisfy $A V-V A=0$. Then $B-(A V-V A)=B$ and so the partial isometries $U_{1}$ and $U$ in the polar decompositions of $B-(A V-V A)$ and $B$ coincide. Thus, $Y\left(=|B-(A V-V A)|^{p-1} U_{1}^{*}\right)=|B|^{p-1} U^{*} \in \mathscr{C}_{1}$. As in part (a), using Fuglede, we have $|B| A U^{*}=|B| U^{*} A$ and $A|B|^{p-1}=|B|^{p-1} A$. Hence, $\operatorname{Ran}\left(A U^{*}-U^{*} A\right) \subseteq \operatorname{Ker}|B|=\operatorname{Ker}|B|^{p-1}$. So,

$$
A Y-Y A=A|B|^{p-1} U^{*}-|B|^{p-1} U^{*} A=|B|^{p-1}\left(A U^{*}-U^{*} A\right)=0 .
$$

So, as $Y S \in \mathscr{C}_{1}$, then (cf. (4), (3), and (2)) $D_{V} F_{p}(S)=0$ for all $S$ in $\mathscr{L}(H)$.

(c) For $0<p \leq 1$, the finite-dimensionality and invertibility conditions ensure, by Theorem 2.1(b), that $F_{p}$ is differentiable at $V$. If $A V-V A=0$ then $B$, and hence $|B|$, is invertible and so $|B|^{p-1}$ exists for $0<p \leq 1$. The proof of the implication, $A V-V A=0 \Rightarrow V$ is a critical point of $F_{p}$, is now the same as in part (a).

We make some comments.

(i) In Theorem 3.2(a) if $B=A X_{1}-X_{1} A$ for some operator $X_{1}$ then the minimum of $\|B-(A X-X A)\|_{p}$ is 0 . This does not conflict with Theorem 3.2(a) (i.e. (1.3)) because in this case $B=0$; for since the normal operator $A$ commutes with $B\left(=A X_{1}-X_{1} A\right)$ then, by Lemma 3.1, $A X_{1}-X_{1} A=0$.

(ii) The following counterexample shows that Theorem 3.2(a) does not hold if $p<1$. Take $p=\frac{1}{2}$ and $A=\left(\begin{array}{ll}a & 0 \\ 0 & 0\end{array}\right), B=\left(\begin{array}{ll}1 & 0 \\ 0 & 1\end{array}\right)$, and $X=\left(\begin{array}{cc}0 & -x \\ x & 0\end{array}\right)$ where $a$ and $x$ are reals such that $0<|a x|<1$. Then $\|B-(A X-X A)\|_{1 / 2}<\|B\|_{1 / 2}$.

(iii) The set $\mathscr{S}\left(=\left\{X: A X-X A \in \mathscr{C}_{p}\right\}\right)$ properly contains $\mathscr{C}_{p}$, for if $X \in \mathscr{C}_{p}$ then $X \in \mathscr{S}$ and, e.g., $I \in \mathscr{S}$ but $I \notin \mathscr{C}_{p}$. If $A \in \mathscr{C}_{p}$, the conclusions of Theorem 3.2 hold for all $X$ in $\mathscr{L}(H)$.

(iv) The converse in Theorem 3.2(b) can be proved on the basis of the global result: if $A V-V A=0$ then, by Theorem 3.2(a), $V$ is a global minimizer, and hence a critical point, of $F_{p}$.

(v) The proof in Theorem 3.2(b) of the implication, $V$ is a critical point of $F_{p} \Rightarrow A V-V A=0$, does not work in the $0<p \leq 1$ case because the functional calculus argument involving the function $f: t \rightarrow t^{1 /(p-1)}$, where $0 \leq t<\infty$, is only valid for $1<p<\infty$.

(vi) Finally, Anderson's original result (1.2), in the special case where $B$ is compact, can be obtained similarly to Theorem 3.2(a). (Proof: using the fact that $\|S\| \geq \sup _{\|\phi\|=1}|\langle S \phi, \phi\rangle|$, where $S \in \mathscr{L}(H)$, with the basis $\left\{\phi_{n}\right\}=$ $\left\{\xi_{k}\right\} \cup\left\{\psi_{m}\right\}$ as defined in Theorem 3.2(a), we get

$$
\begin{aligned}
\|B-(A X-X A)\| & \geq \sup _{n}\left|\left\langle\left[|B|-U^{*}(A X-X A)\right] \phi_{n}, \phi_{n}\right\rangle\right| \\
& =\sup _{k, m}\left[\left\langle B \mid \xi_{k}, \xi_{k}\right\rangle+\left|\left\langle U^{*}(A X-X A) \psi_{m}, \psi_{m}\right\rangle\right|\right] \\
& \left.\geq \sup _{k}\left\langle|B| \xi_{k}, \xi_{k}\right\rangle=\||B|\|=\|B\| .\right)
\end{aligned}
$$

\section{ACKNOWLEDGMENT}

This paper originated as part of my Ph.D. thesis. I would like to thank my supervisor, Dr. J. A. Erdos, for the help he has so freely given. I would also 
like to thank the referee for drawing my attention to Anderson's paper and for suggesting a strengthening of my original version of Theorem 3.2(a).

\section{REFERENCES}

1. J. G. Aiken, J. A. Erdos, and J. A. Goldstein, Unitary approximation of positive operators, Illinois J. Math. 24 (1980), 61-72.

2. J. Anderson, On normal derivations, Proc. Amer. Math. Soc. 38 (1973), 135-140.

3. N. Dunford and J. T. Schwartz, Linear operators, Part II, Interscience, New York, 1964.

4. P. R. Halmos, Commutators of operators. II, Amer. J. Math. 76 (1954), 191-198.

5. __, Positive approximants of operators, Indiana Univ. Math. J. 21 (1972), 951-960.

6. __ A Hilbert space problem book, 2nd ed., Springer-Verlag, New York, 1974.

7. P. J. Maher, Partially isometric approximation of positive operators, Illinois J. Math. 33 (1989), 227-243.

8. J. R. Ringrose, Compact non-self-adjoint operators, Van Nostrand Rheinhold, London, 1971.

9. H. Wielandt, Ueber die unbeschranktheit der operatoren des quantenmechanik, Math. Ann. 121 (1949), 21.

10. A. Wintner, The unboundedness of quantum-mechanical matrices, Phys. Rev. 71 (1947), 738-739.

School of Mathematics, Middlesex Polytechnic, Trent Park, Bramley Road, London N14 4XS, ENGLAND 